\title{
Inhibition of NF-кB activation by BAY 11-7821 suppresses the proliferation and inflammation of glioma cells through inducing autophagy
}

\author{
Kui Xie ${ }^{1}$, Deming Zhou ${ }^{2}$, Chao Fang ${ }^{3}$, Rong Pu ${ }^{4}$, Zhanpeng Zhu ${ }^{1}$ \\ ${ }^{1}$ Department of Neurosurgery, SSL Central Hospital of Dongguan City, Affiliated Dongguan Shilong People's Hospital of Southern Medical \\ University, Dongguan, China; ${ }^{2}$ Department of Radiology, SSL Central Hospital of Dongguan City, Affiliated Dongguan Shilong People's Hospital \\ of Southern Medical University, Dongguan, China; ${ }^{3}$ Department of Neurosurgery, The Second Hospital of Shenzhen City, Shenzhen, China; \\ ${ }^{4}$ Department of Clinical Laboratory, SSL Central Hospital of Dongguan City, Affiliated Dongguan Shilong People's Hospital of Southern Medical \\ University, Dongguan, China \\ Contributions: (I) Conception and design: K Xie, D Zhou; (II) Administrative support: K Xie; (III) Provision of study materials or patients: D Zhou, C \\ Fang; (IV) Collection and assembly of data: D Zhou, C Fang; (V) Data analysis and interpretation: K Xie, R Pu, Z Zhu; (VI) Manuscript writing: All \\ authors; (VII) Final approval of manuscript: All authors. \\ Correspondence to: Kui Xie. Department of Neurosurgery, SSL Central Hospital of Dongguan City, No. 1 Xianglong Road, Dongguan 523326, China. \\ Email: 583677607@qq.com.
}

\begin{abstract}
Background: Gliomas have been known as the most common intracranial malignant tumor, and this kind of tumors cause huge amounts of mortality. The NF- $\mathrm{kB}$ inhibitor BAY 11-7821 has been reported as a novel approach in the immunotherapy of lung diseases. However, the functional role of BAY 11-7821 and its association with autophagy in glioma cells have not yet been reported.

Methods: In this study, 2 glioma cell lines (U87 and U251) were treated with different doses of BAY 11-7821, or combined with authphagy inhibitor, 3-MA. Afterwards, Transwell assay, CCK-8 assay, EdU staining, Western blot and immunofluorescence assay was used to detected the cell migration, invasion, vability, autophagy in U87 and U251.
\end{abstract}

Results: Our data showed that BAY 11-7821 significantly suppressed the viability, proliferation, migration, and invasion of glioma cells in a dose-dependent manner. At the molecular level, BAY 11-7821 downregulated the protein levels of $\mathrm{p}-\mathrm{I} \kappa \mathrm{B} \alpha, \mathrm{p}-\mathrm{p} 65, \mathrm{NLRP} 3$, and p62, and upregulated the protein levels of caspase 3 and Bax, as well as decreased the levels of IL-1 $\beta$ and IL-18. Results showed BAY 11-7821 enhanced autophagy. While, Pre-treatment with 3-MA, an autophagy inhibitor, obviously reversed the effects of BAY 11-7821 on malignant biological behaviors of glioma cell, inflammation status, and autophagy.

Conclusions: In this study, we found that BAY 11-7821 has an effective inhibitive function on malignant biological behaviors by mediating autophagy. Our findings contribute to a better understanding of BAY 117821 as a potential anticancer drug in glioma via activating autophagy.

Keywords: Glioma; NF-кB; BAY 11-7821; autophagy; inflammation

Submitted Dec 09, 2021. Accepted for publication Jan 30, 2022.

doi: $10.21037 /$ tcr-21-2914

View this article at: https://dx.doi.org/10.21037/tcr-21-2914

\section{Introduction}

Glioma is the most prevalent type of malignant central nervous system cancer, accounting for $70-80 \%$ of malignant brain tumors $(1,2)$. Upon diagnosis, standard treatments, including maximal surgical resection, radiotherapy, and chemotherapy with temozolomide, are applied for malignant glioma (3). Despite achievements in these treatments, the median overall survival for glioma patients is $1-2$ years and the 5 -year survival rate remains less than 
$6 \%$ (4). For the purpose of achieving better outcomes, a better understanding of the underlying molecular mechanisms driving the pathogenesis of glioma is urgently needed.

Autophagy is a conservative cellular pathway that can deactivate damaged organelles and aggregate proteins to promote self-digestion processes (5). The enhancing effects of autophagy on cell survival or cell death makes the role of autophagy in the development of cancer controversial (6). In recent years, autophagy has been used as a new target for the treatment of tumors and even gliomas. For example, arctigenin inhibited the proliferation of glioma cells by inducing autophagy and apoptosis (7). Luteolin may be a potential drug for glioma treatment by suppressing tumor proliferation via inducing apoptosis and autophagy in glioma (8). Hydroxychloroquine is a novel strategy that may augment the efficacy of bevacizumab for glioma via the inhibition of autophagy (9).

$\mathrm{NF}-\kappa \mathrm{B}$, consisting of 3 subunits (p50, p65, and IкB $\alpha$ ), has been widely considered as a key regulator implicated in inflammatory responses and tumorigenesis $(10,11)$. Nodlike receptor family pyrin domain containing 3 (NLRP3), is a component of the NLRP3 inflammasome, participating in the occurrence of many diseases, including human cancers $(12,13)$. A recent study by Xue et al. (14) showed that NLRP3 promoted the growth and invasion of gliomas via IL-1 $1 / \mathrm{NF}-\mathrm{\kappa B}$ p 65 signaling. Autophagy may also modulate the production and secretion of inflammatory cytokines production in tumor cells via diverse mechanisms through complex mechanisms $(15,16)$.

In fact, the relationship between NF- $\mathrm{BB}$ and autophagy is very complicated, since NF- $\kappa B$ participates physiological or pathological activities through many pathways. A study in renal injury discover that the activation of autophagy can inhibit NF- $\kappa \mathrm{B}$ pathway, down-regulate the levels of inflammatory factors, such as IL-1 $\beta$, IL- 6 and TNF- $\alpha$, and reduce the inflammatory response caused by renal injury (17). However, Zhu et al. find that olanzapine induces autophagy and promotes apoptosis of glioma cells by inactivate NF- $\mathrm{KB}$ pathway (18). Study has also confirmed that NF- $\kappa \mathrm{B}$-mediated inflammatory response can be synergistic with autophagy. For example, Li et al. found that in lung injury, DAPK1 triggers inflammatory response and autophagy through the p38MAPK/NF- $\mathrm{BB}$ pathway, which jointly causes lung injury (19). Therefore, it is necessary to explore the relationship between NF- $\kappa \mathrm{B}$-mediated inflammatory response and autophagy in gliomas.

BAY 11-7821, also known as BAY 11-7082, is a selective

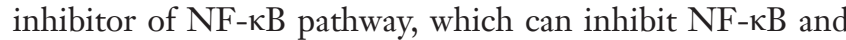
further inhibit inflammasome mediated cell pyrogenesis. It also has the effect of anti-tumor. Wang et al. find that BAY 11-7821 can inhibit cell proliferation and promote apoptosis in multiple myeloma (20). A recent study by Ma et al. (21) showed that when NF- $\mathrm{BB}$ was inhibited by BAY 11-7821, epithelial-mesenchymal transition (EMT) stimulated by cigarette smoke extract (CSE) alone or combined with IL$17 \mathrm{~A}$ was significantly reduced. In fact, BAY 11-7821 is also found to have anti-tumor effect in gliomas. For example, Zhang et al. discover that BAY11-7082 inhibited IL-33induced invasion and migration, and down-regulated the production of MMP2 and MMP9 by blocking the NF- $\mathrm{KB}$ pathway (22). However, the unclear role of BAY11-7082 in gliomas may be one of the reasons why it cannot be widely used.

In this study, we first determined the effects of different doses of BAY 11-7821 on cell proliferation, migration, invasion, inflammation, and autophagy in 2 glioma cell lines (U87 and U251). We further verified that the regulatory role of BAY 11-7821 in glioma cells was related to autophagy. This novel pathway may provide a foundation for the use of BAY 11-7821 as an antitumor intervention. We present the following article in accordance with the MDAR reporting checklist (available at https://tcr. amegroups.com/article/view/10.21037/tcr-21-2914/rc).

\section{Methods}

\section{Cell culture and treatment}

Two glioma cell lines (U87 and U251) were obtained from the Cell Bank of the Chinese Academy of Sciences (Shanghai, China) and cultured in RPMI 1640 medium (Gibco, Grand Island, NY, USA) supplemented with $10 \%$ fetal bovine serum (Gibco) at $37^{\circ} \mathrm{C}$ in a humidified incubator with $5 \% \mathrm{CO}_{2}$. For the in vitro experiments, cells were co-cultured with different doses $(0,2.5,5,10 \mu \mathrm{M})$ of BAY 11-7821 (A4210, APEXBIO, Texas, USA) for 0, 24, 48, and $72 \mathrm{~h}$. Subsequently, the cells were prepared as described for the blank group, BAY group, and BAY + 3-MA group (cells in the high-dose BAY group with $12 \mathrm{~h}$ pre-treatment by the autophagy inhibitor 3-MA).

\section{Cell viability assay}

Glioma cells from different groups were collected and seeded into 96-well plates at a density of 5,000 cells per well. At $0,24,48$, and 72 h, cells were incubated with 
$10 \mu \mathrm{L}$ Cell Counting Kit-8 (CCK-8) solution (Sigma 96992, St. Louis, MO, USA) for $2 \mathrm{~h}$. Afterwards, the absorbance at a wavelength of $450 \mathrm{~nm}$ was measured using a microplate reader (Molecular Devices, Sunnyvale, CA, USA).

\section{5-ethynyl-2'-deoxyuridine (EdU) staining}

The proliferation of glioma cells was assessed by the CellLight EdU DNA Cell Proliferation Kit (RiboBio Co., Ltd, Guangzhou, China) according to the manufacturer's instructions. In brief, cells from different groups were inoculated in 96-well plates in triplicate and fixed with $4 \%$ paraformaldehyde. After permeabilization with $1 \%$ Triton X-100, cells were stained with Alexa-Fluor 594 reaction cocktail for EdU for $30 \mathrm{~min}$. Meanwhile, 4',6-diamidino2-phenylindole (DAPI) was used to stain the cell nuclei for $30 \mathrm{~min}$. The stained images were observed under a fluorescence microscope (Olympus, Japan).

\section{Transwell assay}

The migration and invasion abilities of glioma cells were evaluated using a 24-well transwell chamber (pore size: $8 \mu \mathrm{m}$, EMD Millipore, Billerica, MA, USA) pre-coated with and without $20 \mu \mathrm{L}$ Matrigel (BD Biosciences, San Jose, CA, USA). For the migration and invasion assays, glioma cells $\left(5 \times 10^{4}\right.$ cells $)$ in serum free medium were placed in the upper chamber, and the lower chamber was filled with medium containing $10 \%$ FBS to induce cell migration or invasion. After $24 \mathrm{~h}$ incubation, the migratory or invasive cells in the lower chamber were fixed with $4 \%$ paraformaldehyde and stained with $0.1 \%$ crystal violet. The number of migrated or invasive cells was counted using an inverted light microscope (Zeiss, Oberkochen, Germany).

\section{Enzyme-linked immunosorbent assay (ELISA)}

The concentration levels of IL-1 $\beta$ and IL-18 in the cell supernatants of glioma cells from different groups were determined by highly sensitive ELISA using the specific human IL-1 $\beta$ ELISA kit (CHE0001) and a human IL-18 ELISA kit (CHE0007) purchased from 4A Biotech Co., Ltd., (Beijing, China) according to the manufacturer's instructions.

\section{Western blot analysis}

Total protein samples were obtained using RIPA lysis buffer (Beyotime, Beijing, China) and analyzed for protein concentration with the BCA Protein Assay Kit (Thermo, Rockford, IL, USA). An equal amount of protein was separated by $12 \%$ SDS-PAGE and transferred onto PVDF membranes (Millipore, Billerica, MA, USA). Next, the membranes were blocked with $5 \%$ skim milk for $2 \mathrm{~h}$ and incubated overnight with primary antibodies against I $\mathrm{B} B \alpha$ (ab32518, Abcam Cambridge, UK), p-ІкB $\alpha$ (ab92700, Abcam), p65 (ab16502, Abcam), p-p65 (ab76302, Abcam), NLRP3 (ab263899, Abcam), caspase 3 (ab184787, Abcam), Bax (ab182734, Abcam), LC3B-I/II (ab51520, Abcam), p62 (ab109012, Abcam), and GAPDH (ab181602, Abcam), followed by incubation with horseradish peroxidase conjugated secondary antibody (ab6721, Abcam) for $2 \mathrm{~h}$ at room temperature. The protein bands were visualized using enhanced chemiluminescence (Millipore) and analyzed using Quantity One software (Bio-Rad, CA, USA).

\section{Immunofluorescence staining}

Glioma cells from different groups were seeded into 6-well plates and grown on sterilized coverslips. After fixation by $4 \%$ paraformaldehyde for $30 \mathrm{~min}$, cells were permeabilized with $0.3 \%$ Triton $\mathrm{X}-100$ for $30 \mathrm{~min}$ at $37{ }^{\circ} \mathrm{C}$ and blocked with $5 \%$ bovine serum albumin (BSA) for $1 \mathrm{~h}$. Subsequently, cells were incubated with a primary antibody against LC3B (ab51520, Abcam, Cambridge, UK) at $4{ }^{\circ} \mathrm{C}$ overnight and then incubated with FITC-conjugated anti-rabbit secondary antibody (ab6717, Abcam). Finally, the coverslips were stained with DAPI for $20 \mathrm{~min}$ at $37^{\circ} \mathrm{C}$ and photographed using a fluorescence microscope (Olympus, Japan).

\section{Statistical analysis}

All experiments were technically repeated 3 times. Quantitative data were analyzed using GraphPad Prism 6 software and expressed as mean \pm standard deviation (SD). Differences for multiple comparisons were assessed using one-way analysis of variance (ANOVA) accompanied by Tukey's test. Values of $\mathrm{P}<0.05$ were considered to be statistically significant.

\section{Results}

\section{BAY 11-7821 inbibited the proliferation, migration, and invasion of glioma cells}

Firstly, we investigated the effects of BAY 11-7821 on glioma cellular functions. The results from the CCK8 assay showed that BAY 11-7821 treatment significantly 
A
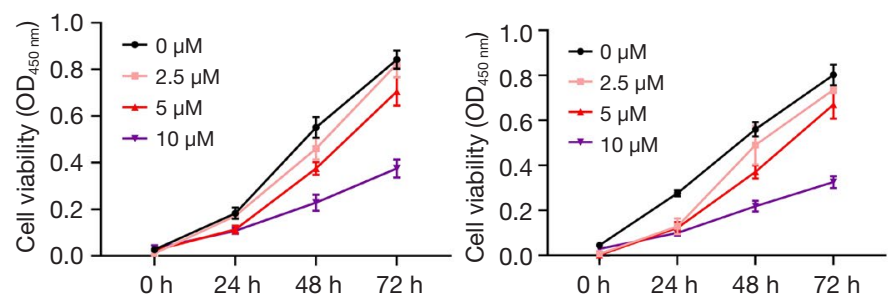

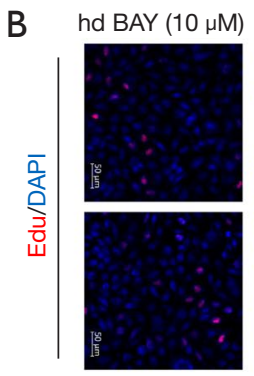

C
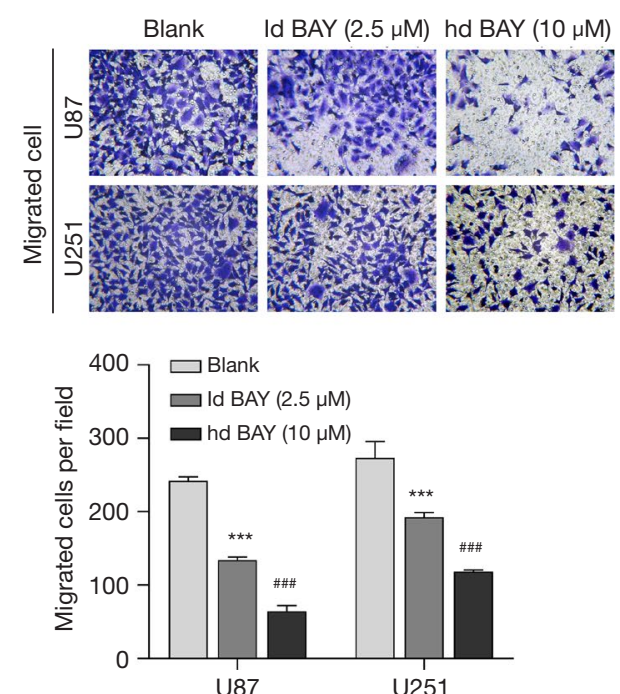

Blank
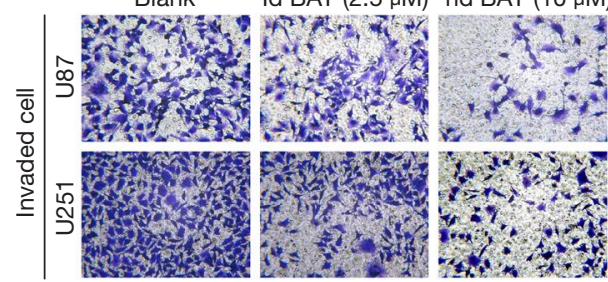

Rida, 2 ?
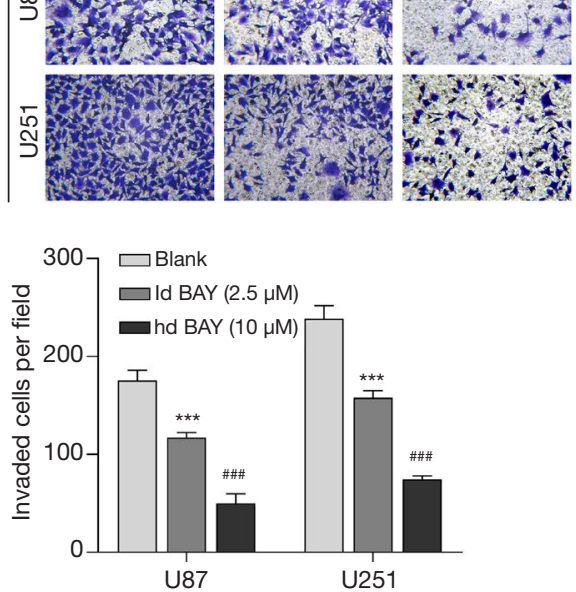

Figure 1 BAY 11-7821 inhibited the proliferation, migration, and invasion of glioma cells. U87 and U251 cells were treated with BAY 11$7821(0,2.5,5,10 \mu \mathrm{M})$ for $0,24,48$, and $72 \mathrm{~h}$. (A) Cell viability was determined in the above treated U87 and U251 cells. (B) The EdU assay was utilized to detect cell proliferation in U87 and U251 cells treated with $2.5 \mu \mathrm{M}$ (low dose) and $10 \mu \mathrm{M}$ (high dose) BAY 11-7821 for 24 h, in which cells were stained with Alexa-Fluor 594 reaction cocktail for EdU and 4',6-diamidino-2-phenylindole (DAPI) was used to stain the cell nuclei (scale: $50 \mu \mathrm{m}$ ). (C) Cell migration and (D) invasion were evaluated using the transwell assay in U87 and U251 cells treated with $2.5 \mu \mathrm{M}$ (low dose) and $10 \mu \mathrm{M}$ (high dose) BAY 11-7821 for $24 \mathrm{~h}$, in which cells in the lower compartment were stained with crystal violet ( $\times 100$ magnification). The above data are measurement data and expressed as the mean $\pm \mathrm{SD}$. ${ }^{* *} \mathrm{P}<0.001$, compared with blank; ${ }^{\# \# \#} \mathrm{P}<0.001$, compared with LD BAY 11-7821. LD, low doses; HD, high doses; BAY, BAY 11-7821; DAPI, 4',6-diamidino-2-phenylindole; Edu, 5-ethynyl-2'-deoxyuridine staining.

impaired cell viability in U87 and U251 cells in a doseand time-dependent manner (Figure 1A). We selected low doses (LD) and high doses (HD) of BAY 11-7821 and $24 \mathrm{~h}$ treatment for the subsequent analysis. The EdU assay demonstrated that BAY 11-7821 treatment decreased the proliferative ability of U87 and U251 cells compared with the blank group (Figure 1B). Furthermore, HD BAY 11-7821 generated stronger suppressive effects on the proliferation of glioma cells. In addition, the number of migrated cells (Figure 1C) and invasive cells (Figure 1D) was markedly decreased in the HD BAY 11-7821 group and slightly reduced in the LD BAY 11-7821 group compared with the blank group.

\section{BAY 11-7821 as an NF-кB inbibitor promoted apoptosis and autophagy in glioma cells}

Considering that NF- $\mathrm{KB}$ signaling plays important roles in regulating inflammatory responses and tumorigenesis, we first determined the effects of BAY 11-7821 on NF- $\kappa \mathrm{B}$ subunits ( $\mathrm{\kappa} \mathrm{B} \alpha, \mathrm{p}-\mathrm{I} \kappa \mathrm{B} \alpha, \mathrm{p} 65, \mathrm{p}-\mathrm{p} 65)$, NLRP3, and pro-inflammatory cytokines (IL-1 $\beta$ and IL-18) in glioma cells. As expected, HD BAY 11-7821, as an NF$\kappa \mathrm{B}$ inhibitor, markedly suppressed the phosphorylation of I $\mathrm{B} \mathrm{B} \alpha$ and $\mathrm{p} 65$ and the NLRP3 protein level in U87 and $\mathrm{U} 251$ cells as determined by western blot analysis (Figure 2A). ELISA consistently showed that HD BAY 11- 
A

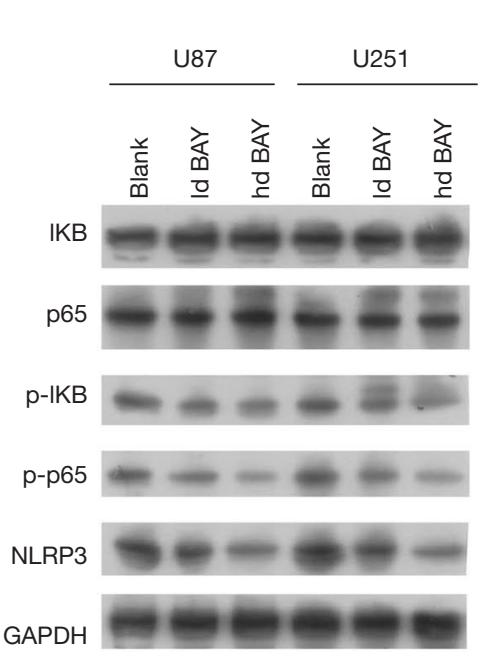

C

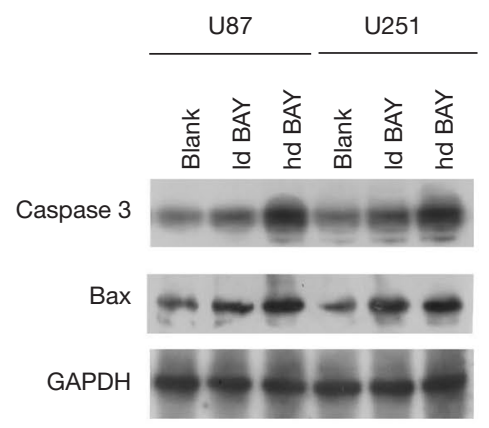

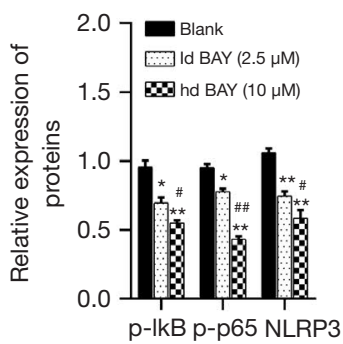

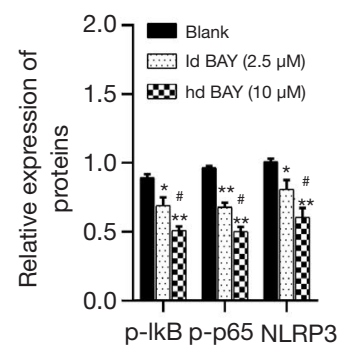

$\mathrm{D}$

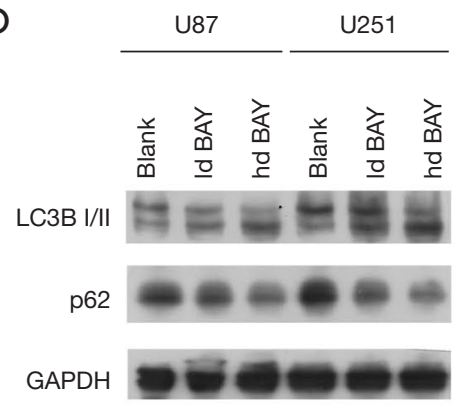

E

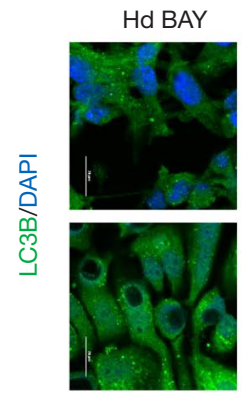

B
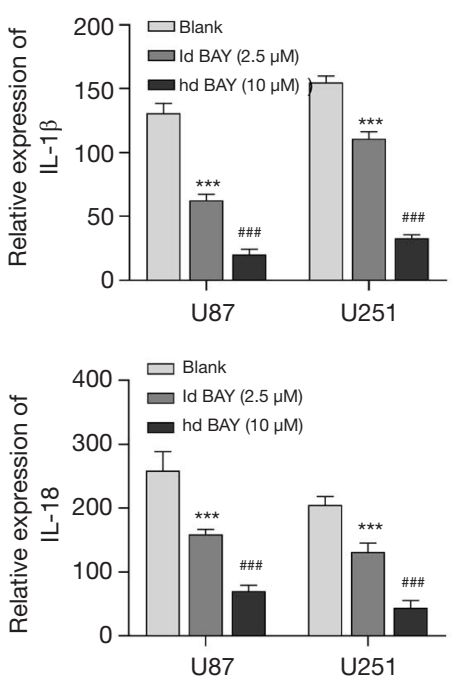
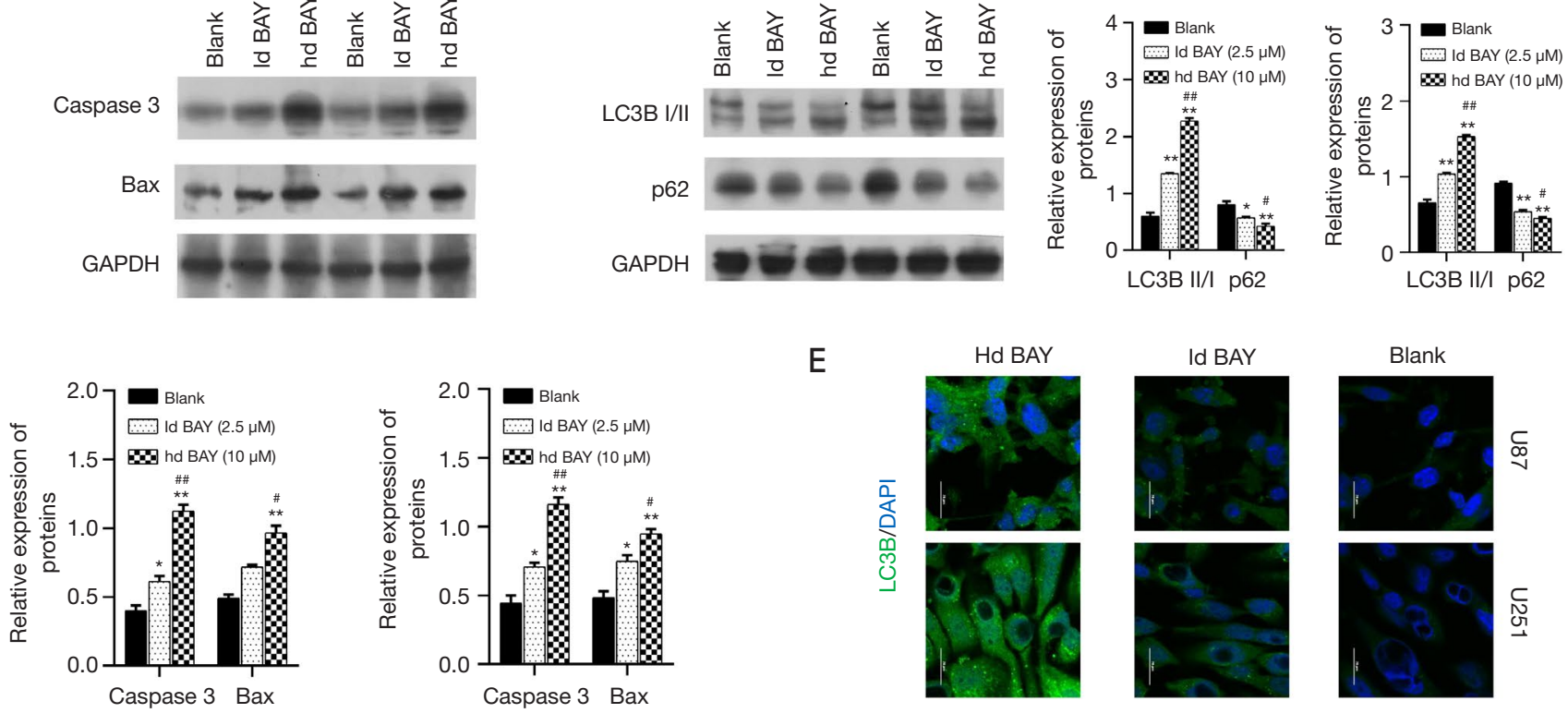

Figure 2 BAY 11-7821 as an NF- $\mathrm{kB}$ inhibitor promoted apoptosis and autophagy in glioma cells. U87 and U251 cells were treated with low doses and high doses of BAY 11-7821. (A) The protein levels of I $\mathrm{KB} \alpha, \mathrm{p}-\mathrm{I} \kappa \mathrm{B} \alpha$, p65, p-p65, and NLRP3 were measured in the above treated glioma cells using western blot analysis; (B) ELISA was performed to analyze the concentration levels of IL-1 $\beta$ and IL-18 in cell supernatants of the above treated glioma cells; (C) the protein levels of caspase 3 and Bax were measured in the above treated glioma cells using western blot analysis; (D) the protein levels of LC3B I/II and p62 were measured in the above treated glioma cells using western blot analysis. The above data are measurement data and expressed as the mean $\pm \mathrm{SD}$. ${ }^{*} \mathrm{P}<0.05,{ }^{* *} \mathrm{P}<0.01,{ }^{* * *} \mathrm{P}<0.001$, compared with blank; ${ }^{\#} \mathrm{P}<0.05,{ }^{\# \#} \mathrm{P}<0.01,{ }^{\# \# !} \mathrm{P}<0.001$, compared with LD BAY 11-7821; (E) immunofluorescence staining of LC3B in the above treated glioma cells, in which cells were incubated with a primary antibody against LC3B, then incubated with FITC-conjugated anti-rabbit secondary antibody, and the coverslips were stained with DAPI (scale: $20 \mu \mathrm{m}$ ). LD, low doses; HD, high doses; BAY, BAY 11-7821; DAPI, 4',6-diamidino-2phenylindole. 
A

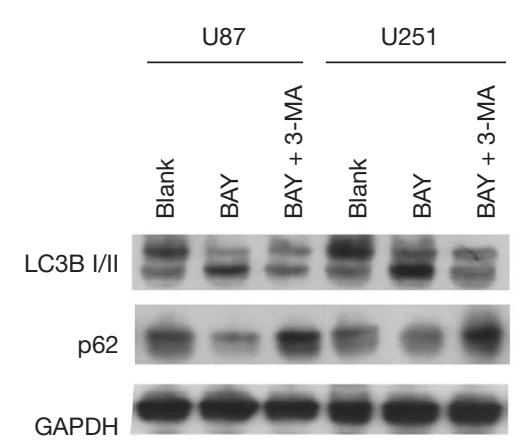

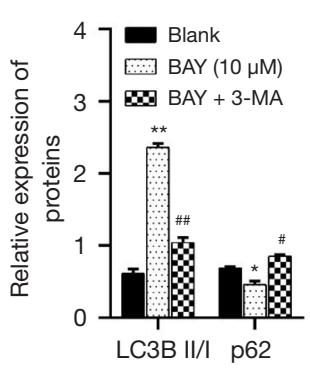

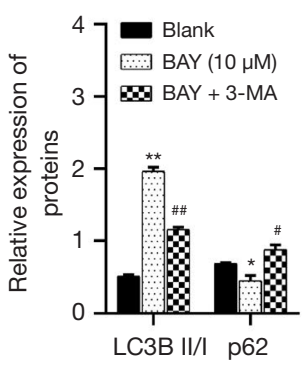

B

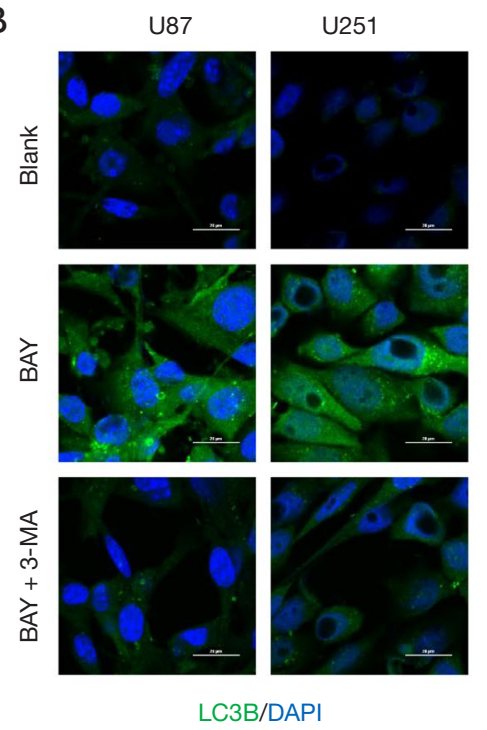

Figure 3 Validation of 3-MA-induced autophagy inhibition in BAY 11-7821 treated glioma cells. BAY 11-7821 was used at $10 \mu M$. (A) The protein levels of LC3B I/II and p62 were measured in U87 and U251 cells from the high-dose BAY 11-7821 group after 3-MA treatment using western blot analysis. (B) Immunofluorescence staining of LC3B in the above treated glioma cells, in which cells were incubated with a primary antibody against LC3B, then incubated with FITC-conjugated anti-rabbit secondary antibody, and the coverslips were stained with DAPI (scale: $20 \mu \mathrm{m}$ ). The above data are measurement data and expressed as the mean $\pm \mathrm{SD} .{ }^{*} \mathrm{P}<0.05,{ }^{* *} \mathrm{P}<0.01$, compared with blank; ${ }^{\#} \mathrm{P}<0.05,{ }^{\# \#} \mathrm{P}<0.01$, compared with BAY 11-7821. BAY, BAY 11-7821; DAPI, 4',6-diamidino-2-phenylindole.

7821 treatment significantly reduced the relative expression of IL-1 $\beta$ and IL-18 in U87 and U251 cells (Figure $2 B$ ). Under the inhibition of NF- $\kappa B$ signaling, we further analyzed whether BAY 11-7821 affected the activation of apoptosis and autophagy. As shown in Figure 2C, HD BAY 11-7821 treatment markedly upregulated the protein levels of pro-apoptotic caspase 3 and Bax in U87 and U251 cells. Moreover, autophagy was markedly activated after HD BAY 11-7821 treatment, as reflected by upregulated LC3B I/ II and downregulated p62 protein expression (Figure 2D), as well as strong immunofluorescence staining of LC3B (Figure 2E) in U87 and U251 cells.

\section{Inhibition of autophagy reversed the suppressive effects of BAY 11-7821 on glioma cell proliferation, migration, and invasion}

To further investigate whether BAY 11-7821 exerted suppressive effects on glioma cellular functions through activating autophagy, 3-MA was used to inhibit the activation of autophagy. Firstly, we confirmed the inhibition of autophagy after 3-MA treatment, as demonstrated by downregulated LC3B I/II and upregulated p62 expression (Figure $3 A$ ), as well as impaired immunofluorescence staining of LC3B (Figure 3B) in U87 and U251 cells from the HD BAY 11-7821 group. Next, we investigated the proliferation, migration, and invasion abilities of glioma cells under 3-MA treatment. The EdU assay indicated that the decrease in EdU-positive cells by HD BAY 11-7821 treatment was obviously elevated under 3-MA treatment (Figure 4A). Similarly, 3-MA treatment significantly reversed the decreased number of migrated and invaded cells induced by HD BAY 11-7821 treatment in both U87 and $\mathrm{U} 251$ cells (Figure 4B).

\section{Inbibition of autophagy reversed the regulatory effect of $B A Y$ 11-7821 on apoptosis and inflammation in glioma cells}

At the molecular level, we first confirmed that 3-MA treatment suppressed the upregulation of caspase 3 and Bax induced by HD BAY 11-7821 treatment in U87 and U251 cells (Figure 5A). Subsequently, we observed that the downregulation of p-I $\mathrm{KB} \alpha, \mathrm{p}-\mathrm{p} 65$, and NLRP3 in U87 and U251 cells in the HD BAY 11-7821 group was notably reversed after inhibition of autophagy via 3-MA treatment (Figure 5B). Additionally, decreased expression levels of IL$1 \beta$ and IL-18 in the HD BAY 11-7821 group were both increased after 3-MA treatment (Figure 5C). These data 
A

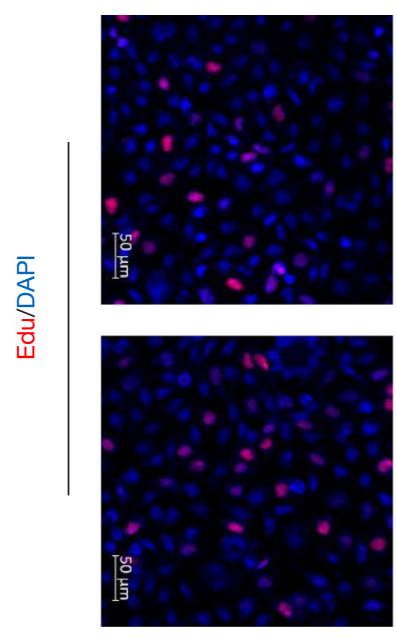

B

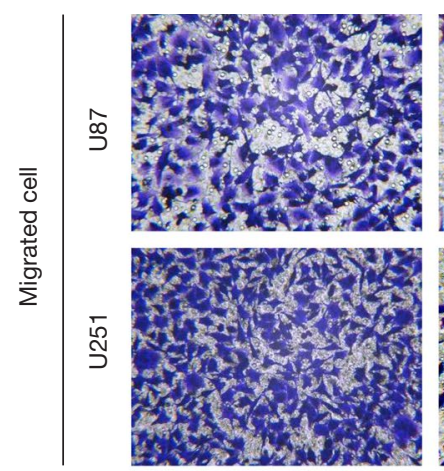

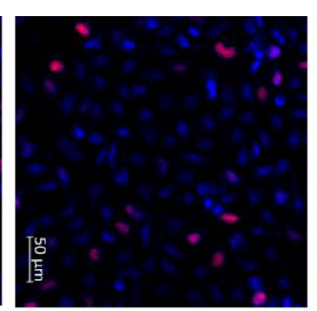
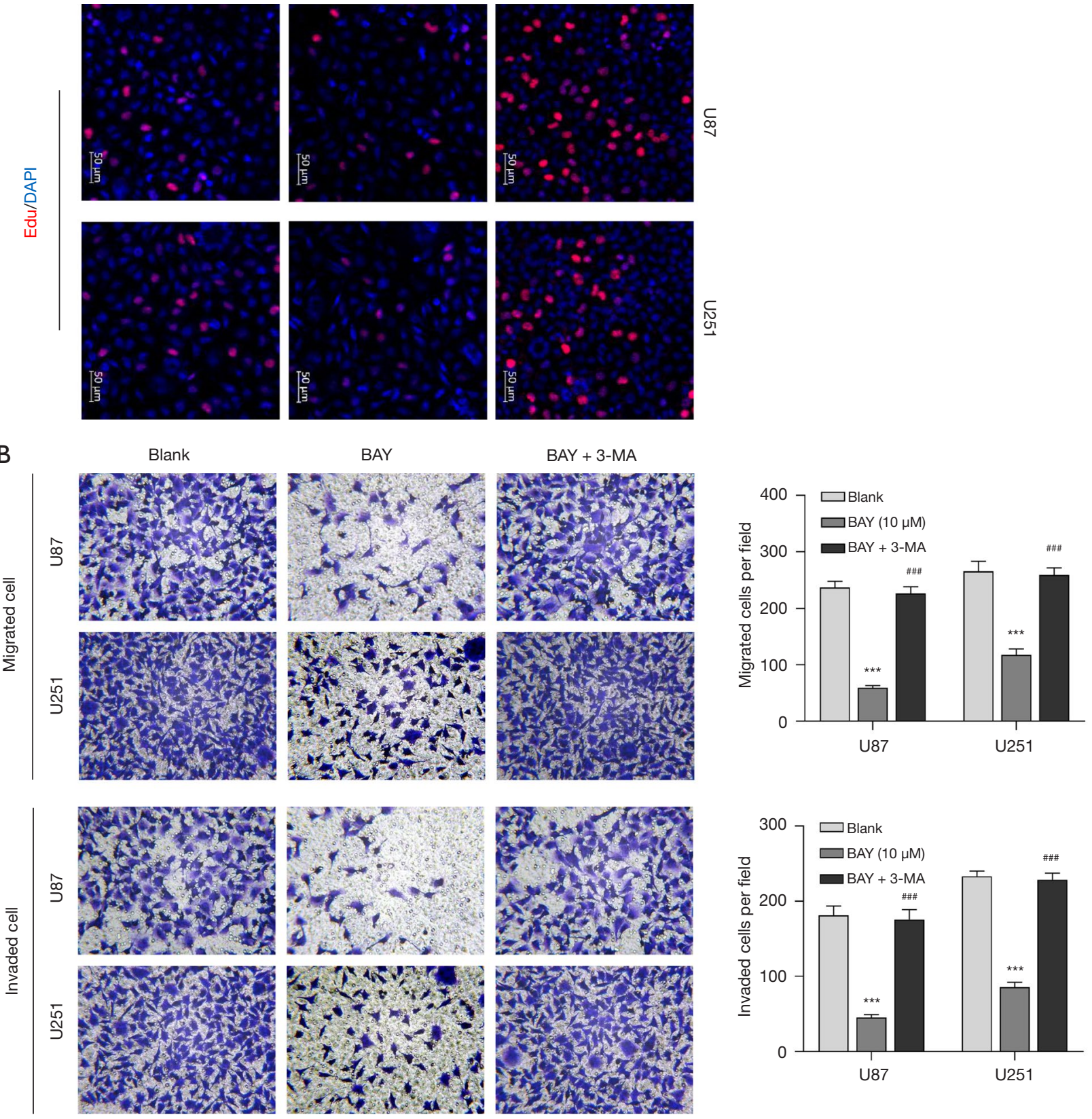

Figure 4 Inhibition of autophagy reversed the suppressive effects of BAY 11-7821 on glioma cell proliferation, migration, and invasion. BAY 11-7821 was used at $10 \mu \mathrm{M}$. (A) The EdU assay was utilized to detect proliferation in U87 and U251 cells from the high-dose BAY 11-7821 group after 3-MA treatment in which cells were stained with Alexa-Fluor 594 reaction cocktail for EdU and 4',6-diamidino-2phenylindole (DAPI) was used to stain the cell nuclei (scale: $50 \mu \mathrm{m}$ ). (B) Cell migration and invasion were evaluated using the transwell assay in U87 and U251 cells from the high-dose BAY 11-7821 group after 3-MA treatment, in which cells in the lower compartment were stained with crystal violet ( $\times 100$ magnification). The above data are measurement data and expressed as the mean $\pm \mathrm{SD} .{ }^{* * *} \mathrm{P}<0.001$, compared with blank; ${ }^{\# \#+} \mathrm{P}<0.001$, compared with BAY 11-7821. BAY, BAY 11-7821; DAPI, 4',6-diamidino-2-phenylindole; Edu, 5-ethynyl-2'-deoxyuridine staining. 
A

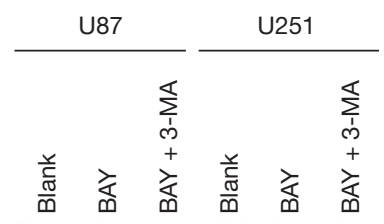

Caspase 3

Bax

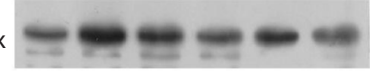

GAPDH

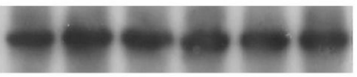

B

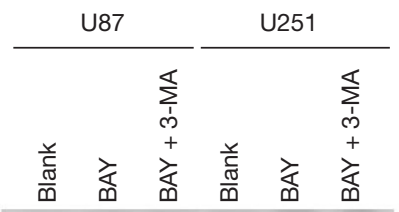

IKB

p65

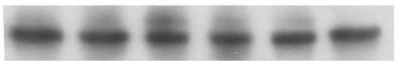

$\mathrm{p}-\mathrm{IKB}$

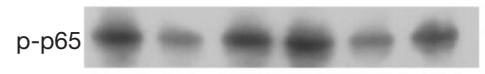

NLRP3

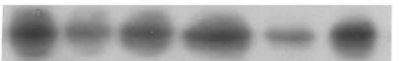

GAPDH
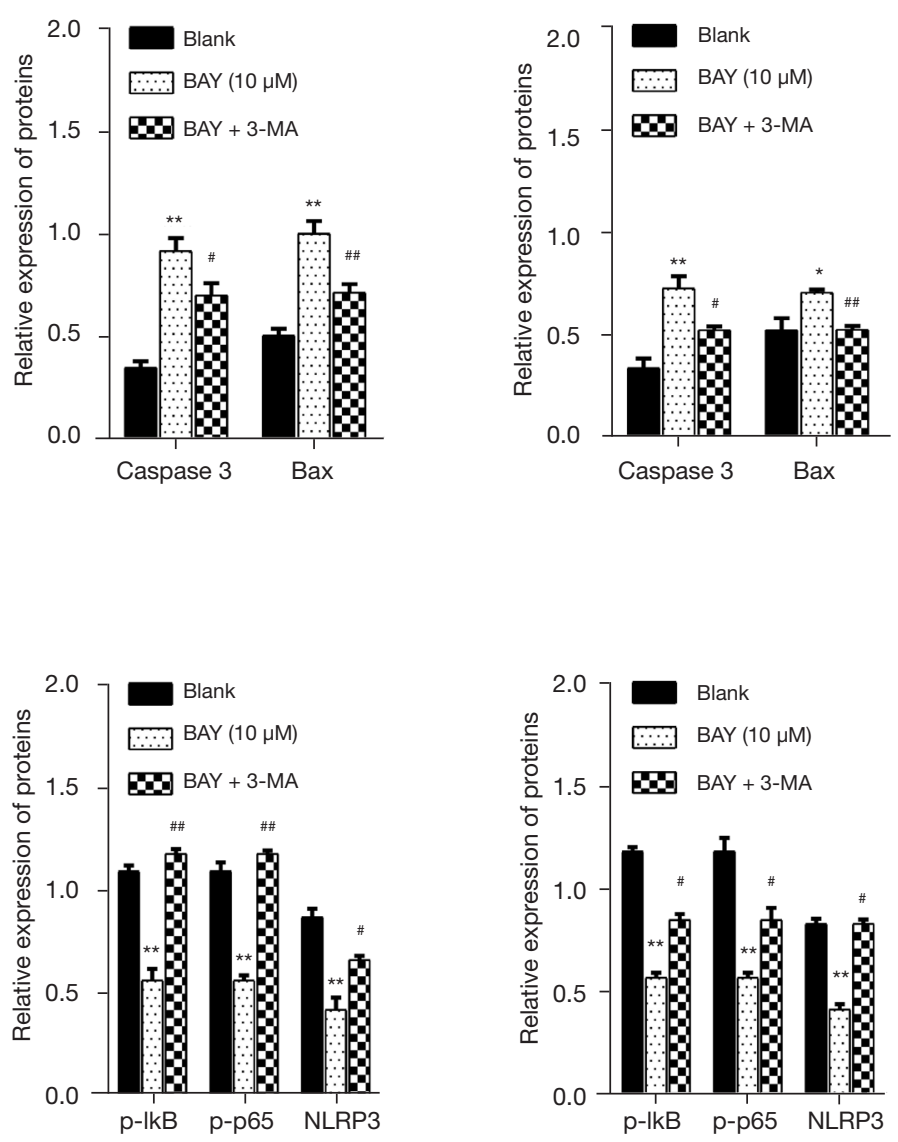

C
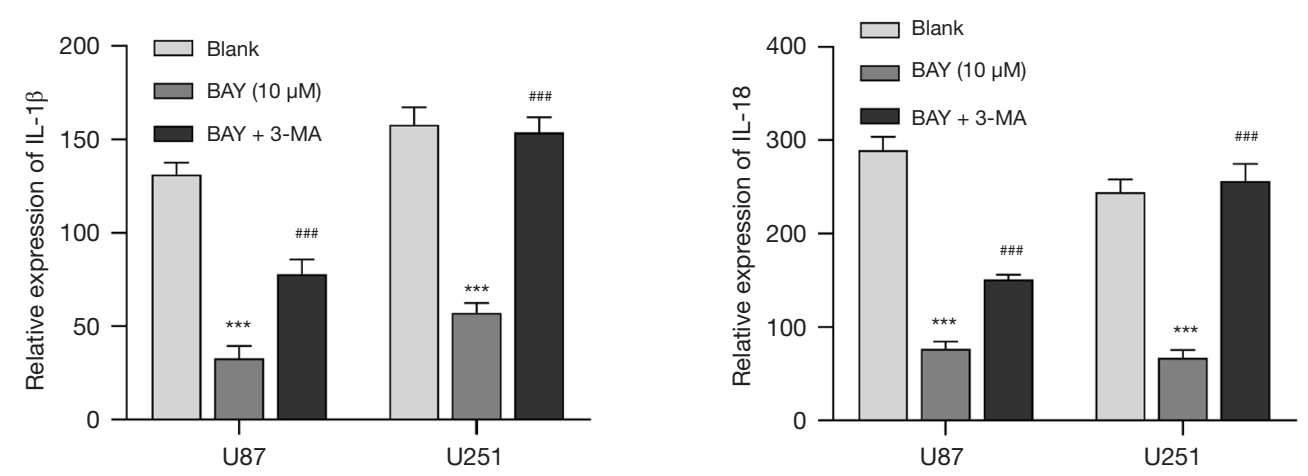

Figure 5 Inhibition of autophagy reversed the regulatory effect of BAY 11-7821 on apoptosis and inflammation in glioma cells. BAY 117821 was used at $10 \mu \mathrm{M}$. U87 and U251 cells from the high-dose BAY 11-7821 group were treated with 3-MA. Western blot analysis was performed to analyze the protein levels of (A) pro-apoptosis related factors (caspase 3 and Bax) and (B) NF- $\kappa \mathrm{B}$ signaling related factors (I $\kappa \mathrm{B} \alpha$, $\mathrm{p}-\mathrm{I} \kappa \mathrm{B} \alpha, \mathrm{p} 65, \mathrm{p}-\mathrm{p} 65, \mathrm{NLRP} 3)$ in the above treated glioma cells. (C) ELISA was performed to analyze the concentration levels of IL-1 $\beta$ and $\mathrm{IL}-18$ in cell supernatants of the above treated glioma cells. The above data are measurement data and expressed as the mean $\pm \mathrm{SD}$. ${ }^{*} \mathrm{P}<0.05$, ${ }^{* *} \mathrm{P}<0.01,{ }^{* * *} \mathrm{P}<0.001$, compared with blank; ${ }^{\#} \mathrm{P}<0.05,{ }^{\# \#} \mathrm{P}<0.01,{ }^{\# \# *} \mathrm{P}<0.001$, compared with BAY 11-7821. BAY, BAY 11-7821. 
indicate that BAY 11-7821-induced NF- $\kappa \mathrm{B}$ inhibition stimulates autophagy to suppress glioma cell functions and inflammatory response.

\section{Discussion}

The present study reports on the antitumor effect of BAY 11-7821 in vitro. We first observed that high doses of BAY 11-7821 treatment had inhibitory effects on the proliferation, migration, and invasion abilities of glioma cells. After confirming the inhibition of NF- $\mathrm{KB}$ signaling by BAY 11-7821, we further found that high doses of BAY 11-7821 treatment suppressed pro-inflammatory cytokines and promoted pro-apoptotic factors. Activation of the NF$\kappa \mathrm{B}$ pathway and NLRP3 inflammasome has been widely reported to increase tumorigenicity in glioma progression. For example, TRIM31 promoted glioma proliferation and invasion through activating NF- $\mathrm{BB}$ activity (21). NLRP3 silencing remarkably impeded the malignant biological properties of glioma cell lines and decreased IL-1 $\beta$ and NF- $\kappa B$ p65 expression (14). NLRP3 significantly elevated IL-1 $\beta$, promoted cell viability, migration/invasion inhibited apoptosis by activating AKT pathway in glioma cells (23). Consistent with our data, miR-98 could promote the apoptosis of glioma cells via inhibiting IKBKE/NF- $\kappa B$ pathway (24). Inhibition of the NF- $\mathrm{NB}$ signaling pathway by CUEDC2 expression suppressed glioma cell proliferation, migration, and invasion (25). In addition, as a marker of inflammation, NF- $\kappa B$ has always been a focus issue in the study of inflammation-induced cancer (26). As reported by Waters et al. (27), glioma cells continuously secrete pro-inflammatory cytokines and factors which attract and activate glioma-associated microglia/macrophages and thus promote a feedforward inflammatory loop. Our data further supported that BAY 11-7821 decreased the inflammatory response via suppressing the NF- $\mathrm{\kappa B}$ signaling pathway and NLRP3 inflammasome.

Notably, we found that BAY 11-7821 treatment activated autophagy in glioma cells, as reflected by downregulated LC3B I/II and upregulated p62 expression. The rescue experiments showed that 3-MA treatment significantly reversed the regulatory effect of BAY 11-7821 on apoptosis and inflammation in glioma cells. These findings suggested that activation of autophagy might be an important process involved in the suppressive effects of BAY 11-7821 on glioma cell proliferation and inflammation. As a matter of fact, inflammation has a complex effect on autophagy in a context-dependent manner (28). In fact, NF- $\kappa \mathrm{B}$ signaling interacts with autophagy to alter tumor cell survival and apoptosis (29). In line with our data, Su et al. (30) demonstrated that sulfasalazine (SAS), a classic inhibitor of NF- $\kappa \mathrm{B}$, leads an up-regulation of apoptosis in a p62dependent manner in U251 cells. According to an updated report by Ma et al. (21), BAY 11-7821 could significantly inhibit the expression of E-cadherin and vimentin in bronchial epithelial cells after concomitant treatment with CSE and IL-17A. In this study, BAY 11-7821 induced the activation of autophagy, which was an important mechanism underlying the tumor suppressive effects of BAY 11-7821 on glioma cells in vitro. Lethal autophagy and protective autophagy have been identified as novel therapeutic targets in glioma, driven by different mechanisms (31). Based on our data, we therefore speculated that BAY 11-7821-induced autophagy is a form of protective autophagy under the inhibition of the NF- $\mathrm{KB}$ signaling pathway in glioma.

In conclusion, results suggested that BAY 11-7821 activates autophagy by suppressing NF- $\mathrm{kB}$, resulting in autophagic cell death and inhibiting glioma cell malignant biological properties, and inflammation. The identification of this pathway enhances our understanding of BAY 117821 as a novel anticancer drug.

\section{Acknowledgments}

Funding: This study was supported by a grant from Medical Scientific Research Foundation of Guangdong Province of China (A2019171).

\section{Footnote}

Reporting Checklist: The authors have completed the MDAR reporting checklist. Available at https://tcr.amegroups.com/ article/view/10.21037/tcr-21-2914/rc

Data Sharing Statement: Available at https://tcr.amegroups. com/article/view/10.21037/tcr-21-2914/dss

Conflicts of Interest: All authors have completed the ICMJE uniform disclosure form (available at https://tcr.amegroups. com/article/view/10.21037/tcr-21-2914/coif). The authors have no conflicts of interest to declare.

Ethical Statement: The authors are accountable for all aspects of the work in ensuring that questions related to the accuracy or integrity of any part of the work are appropriately investigated and resolved. 
Open Access Statement: This is an Open Access article distributed in accordance with the Creative Commons Attribution-NonCommercial-NoDerivs 4.0 International License (CC BY-NC-ND 4.0), which permits the noncommercial replication and distribution of the article with the strict proviso that no changes or edits are made and the original work is properly cited (including links to both the formal publication through the relevant DOI and the license). See: https://creativecommons.org/licenses/by-nc-nd/4.0/.

\section{References}

1. Ohgaki H. Epidemiology of brain tumors. Methods Mol Biol 2009;472:323-42.

2. Ostrom QT, Gittleman H, Farah P, et al. CBTRUS statistical report: Primary brain and central nervous system tumors diagnosed in the United States in 2006-2010. Neuro Oncol 2013;15 Suppl 2:ii1-56.

3. Linz U. Commentary on Effects of radiotherapy with concomitant and adjuvant temozolomide versus radiotherapy alone on survival in glioblastoma in a randomised phase III study: 5-year analysis of the EORTC-NCIC trial (Lancet Oncol. 2009;10:459-466). Cancer 2010;116:1844-6.

4. Shergalis A, Bankhead A 3rd, Luesakul U, et al. Current Challenges and Opportunities in Treating Glioblastoma. Pharmacol Rev 2018;70:412-45.

5. Mizushima N, Levine B, Cuervo AM, et al. Autophagy fights disease through cellular self-digestion. Nature 2008;451:1069-75.

6. Shintani T, Klionsky DJ. Autophagy in health and disease: a double-edged sword. Science 2004;306:990-5.

7. Jiang Y, Liu J, Hong W, et al. Arctigenin Inhibits Glioblastoma Proliferation through the AKT/mTOR Pathway and Induces Autophagy. Biomed Res Int 2020;2020:3542613.

8. You Y, Wang R, Shao N, et al. Luteolin suppresses tumor proliferation through inducing apoptosis and autophagy via MAPK activation in glioma. Onco Targets Ther 2019;12:2383-96.

9. Liu LQ, Wang SB, Shao YF, et al. Hydroxychloroquine potentiates the anti-cancer effect of bevacizumab on glioblastoma via the inhibition of autophagy. Biomed Pharmacother 2019;118:109339.

10. Ben-Neriah Y, Karin M. Inflammation meets cancer, with NF- $\mathrm{KB}$ as the matchmaker. Nat Immunol 2011;12:715-23.

11. Karin M, Greten FR. NF-kappaB: linking inflammation and immunity to cancer development and progression. Nat
Rev Immunol 2005;5:749-59.

12. Bae JY, Lee SW, Shin YH, et al. P2X7 receptor and NLRP3 inflammasome activation in head and neck cancer. Oncotarget 2017;8:48972-82.

13. Feng X, Luo Q, Zhang H, et al. The role of NLRP3 inflammasome in 5 -fluorouracil resistance of oral squamous cell carcinoma. J Exp Clin Cancer Res 2017;36:81.

14. Xue L, Lu B, Gao B, et al. NLRP3 Promotes Glioma Cell Proliferation and Invasion via the Interleukin-1 $\beta / \mathrm{NF}-\kappa \mathrm{B}$ p65 Signals. Oncol Res 2019;27:557-64.

15. Crişan TO, Plantinga TS, van de Veerdonk FL, et al. Inflammasome-independent modulation of cytokine response by autophagy in human cells. PLoS One 2011;6:e18666.

16. Lock R, Kenific CM, Leidal AM, et al. Autophagydependent production of secreted factors facilitates oncogenic RAS-driven invasion. Cancer Discov 2014;4:466-79.

17. Peng X, Wang $\mathrm{Y}, \mathrm{Li} \mathrm{H}$, et al. ATG5-mediated autophagy suppresses NF- $\mathrm{BB}$ signaling to limit epithelial inflammatory response to kidney injury. Cell Death Dis 2019;10:253.

18. Zhu Y, Zhao YF, Liu RS, et al. Olanzapine induced autophagy through suppression of NF- $\mathrm{kB}$ activation in human glioma cells. CNS Neurosci Ther 2019;25:911-21.

19. Li T, Wu YN, Wang H, et al. Dapk1 improves inflammation, oxidative stress and autophagy in LPSinduced acute lung injury via $\mathrm{p} 38 \mathrm{MAPK} / \mathrm{NF}-\kappa \mathrm{B}$ signaling pathway. Mol Immunol 2020;120:13-22.

20. Wang Y, Zhang XL, Sun CM. BAY-11-7082 induces apoptosis of multiple myeloma U266 cells through inhibiting NF- $\kappa B$ pathway. Eur Rev Med Pharmacol Sci 2018;22:2564-71.

21. Ma L, Jiang M, Zhao X, et al. Cigarette and IL-17A synergistically induce bronchial epithelial-mesenchymal transition via activating IL-17R/NF- $\kappa \mathrm{B}$ signaling. BMC Pulm Med 2020;20:26.

22. Zhang JF, Wang P, Yan YJ, et al. IL-33 enhances glioma cell migration and invasion by upregulation of MMP2 and MMP9 via the ST2-NF- $\mathrm{BB}$ pathway. Oncol Rep 2017;38:2033-42.

23. Yin XF, Zhang Q, Chen ZY, et al. NLRP3 in human glioma is correlated with increased WHO grade, and regulates cellular proliferation, apoptosis and metastasis via epithelial-mesenchymal transition and the PTEN/AKT signaling pathway. Int J Oncol 2018;53:973-86.

24. Wang L, Guo S, Zhang H. MiR-98 Promotes Apoptosis 
of Glioma Cells via Suppressing IKBKE/NF-кB Pathway. Technol Cancer Res Treat 2017;16:1226-34.

25. Li F, Tang C, Jin D, et al. CUEDC2 suppresses glioma tumorigenicity by inhibiting the activation of STAT3 and NF- $\mathrm{B}$ signaling pathway. Int J Oncol 2017;51:115-27.

26. Karin M, Cao Y, Greten FR, et al. NF-kappaB in cancer: from innocent bystander to major culprit. Nat Rev Cancer 2002;2:301-10.

27. Waters MR, Gupta AS, Mockenhaupt K, et al. RelB acts as a molecular switch driving chronic inflammation in glioblastoma multiforme. Oncogenesis 2019;8:37.

28. Monkkonen T, Debnath J. Inflammatory signaling cascades and autophagy in cancer. Autophagy 2018;14:190-8.

Cite this article as: Xie K, Zhou D, Fang C, Pu R, Zhu Z. Inhibition of NF- $\mathrm{BB}$ activation by BAY 11-7821 suppresses the proliferation and inflammation of glioma cells through inducing autophagy. Transl Cancer Res 2022;11(2):403-413. doi: 10.21037/ tcr-21-2914
29. Martinez-Outschoorn UE, Whitaker-Menezes D, Lin $Z$, et al. Cytokine production and inflammation drive autophagy in the tumor microenvironment: role of stromal caveolin-1 as a key regulator. Cell Cycle 2011;10:1784-93.

30. Su J, Liu F, Xia M, et al. p62 participates in the inhibition of NF- $\kappa \mathrm{B}$ signaling and apoptosis induced by sulfasalazine in human glioma U251 cells. Oncol Rep 2015;34:235-43.

31. Feng F, Zhang M, Yang C, et al. The dual roles of autophagy in gliomagenesis and clinical therapy strategies based on autophagic regulation mechanisms. Biomed Pharmacother 2019;120:109441.

(English Language Editor: C. Betlazar-Maseh) 